\title{
Modelling scattering and resonances of weakly-bound radioactive nuclei
}

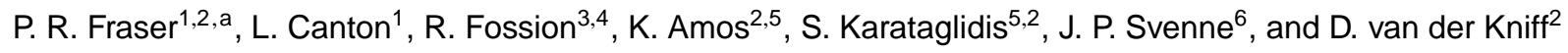 \\ ${ }^{1}$ Istituto Nazionale di Fisica Nucleare, Sezione di Padova, Padova I-35131, Italia \\ ${ }^{2}$ School of Physics, University of Melbourne, Victoria 3010, Australia \\ ${ }^{3}$ Instituto Nacional de Geriatría, Periférico Sur No. 2767, Col. San Jerónimo Lídice, Del. Magdalena Contreras, 10200 Méx- \\ ico D.F., Mexico \\ ${ }^{4}$ Centro de Ciencias de la Complejidad (C3), Universidad Nacional Autónoma de México, 04510 México D.F., Mexico \\ ${ }^{5}$ Department of Physics, University of Johannesburg, P.O. Box 524 Auckland Park, 2006, South Africa \\ ${ }^{6}$ Department of Physics and Astronomy, University of Manitoba, and Winnipeg Institute for Theoretical Physics, Winnipeg, Mani- \\ toba, Canada R3T 2N2
}

\begin{abstract}
For a theoretical description of weakly-bound systems studied with radioactive ion beams, it is important to make allowance for low-lying collective excited states of the colliding bodies which are not stable levels, but rather particle-emitting resonances. This work describes one such implementation in the framework of a multi-channel algebraic scattering formalism for determining nucleon-nucleus cross sections and spectra at low energies.
\end{abstract}

\section{Introduction}

The study of scattering of quantum bodies is of great importance to modern physics, as it is the only way of experimentally probing physics on this scale. Aside from its own intrinsic interest, the investigation of nuclear scattering processes is essential for other fields of physics, especially the study of astrophysical bodies.

These processes may be described by a set of coupledchannel Lippmann-Schwinger equations. Those are:

$$
\begin{aligned}
T_{c c^{\prime}}^{J^{\pi}}(p, q ; E)=V_{c c^{\prime}}^{J^{\pi}}(p, q) & \\
+\mu & {\left[\sum_{c^{\prime \prime}=1}^{\text {open }} \int_{0}^{\infty} V_{c c^{\prime \prime}}^{J^{\pi}}(p, x) \frac{x^{2}}{k_{c^{\prime \prime}}^{2}-x^{2}+i \epsilon} T_{c^{\prime \prime} c^{\prime}}^{J^{\pi}}(x, q ; E) d x\right.} \\
& \left.-\sum_{c^{\prime \prime}=1}^{\text {closed }} \int_{0}^{\infty} V_{c c^{\prime \prime}}^{J^{\pi}}(p, x) \frac{x^{2}}{h_{c^{\prime \prime}}^{2}+x^{2}} T_{c^{\prime \prime} c^{\prime}}^{J^{\pi}}(x, q ; E) d x\right]
\end{aligned}
$$

with the wave numbers being

$$
k_{c}=\sqrt{\mu\left(E-\epsilon_{c}\right)} \text { and } h_{c}=\sqrt{\mu\left(\epsilon_{c}-E\right)} .
$$

The Multi-channel Algebraic Scattering (MCAS) method of solving these, thus far for NA scattering, is built upon finite-rank separable representations of realistic nuclear interaction input potentials. This is done using the Hilbert-Schmidt expansion of amplitudes, whereby a separable $T$-matrix leads to separable potentials. This being true in reverse, potentials are expanded via

$$
V_{c c^{\prime}}=-\sum_{p}\left|\chi_{c p}\right\rangle \frac{1}{\eta_{p}}\left\langle\chi_{c^{\prime} p}\right|
$$

yielding solutions to the Lippmann-Schwinger equations

$$
T_{c c^{\prime}}=-\sum_{p}\left|\chi_{c p}\right\rangle \frac{1}{\left[1-\eta_{p}\right] \eta_{p}}\left\langle\chi_{c^{\prime} p}\right|,
$$

The $\left|\chi_{c p}\right\rangle$ are determined from sturmians [1], and $\eta_{p}$ are their eigenvalues. Importantly, these potentials are modified by the addition of orthogonalising pseudo potentials to make them consistent with the prescriptions of the Pauli exclusion principle, even if collective models are used to specify nuclear targets. Full details are in Ref. [2].

The above expansion leads to the $S$-matrix

$$
\begin{aligned}
& S_{c c^{\prime}}=\delta_{c c^{\prime}}- \\
& i^{l_{c^{\prime}}-l_{c}+1} \pi \mu \sum_{n, n^{\prime}=1}^{N} \sqrt{k_{c}} \hat{\chi}_{c n}\left(k_{c}\right)\left(\left[\boldsymbol{\eta}-\mathbf{G}_{0}\right]^{-1}\right)_{n n^{\prime}} \hat{\chi}_{c^{\prime} n^{\prime}}\left(k_{c^{\prime}}\right) \sqrt{k_{c^{\prime}}}
\end{aligned}
$$

where the Green's functions are

$$
\begin{aligned}
{\left[\mathbf{G}_{0}\right]_{n n^{\prime}}=\mu } & {\left[\sum_{c=1}^{\text {open }} \int_{0}^{\infty} \hat{\chi}_{c n}(x) \frac{x^{2}}{k_{c}^{2}-x^{2}+i \epsilon} \hat{\chi}_{c n^{\prime}}(x) d x\right.} \\
& \left.-\sum_{c=1}^{\text {closed }} \int_{0}^{\infty} \hat{\chi}_{c n}(x) \frac{x^{2}}{h_{c}^{2}+x^{2}} \hat{\chi}_{c n^{\prime}}(x) d x\right] .
\end{aligned}
$$

In applications to date, the nuclear target has been of light mass, but higher masses are possible with more channels and thus longer calculation times. Likewise, applications have been restricted to low energy, but this can be expanded, to an extent, by inclusion of more partial waves. MCAS has shown great success for particle-stable targets

\footnotetext{
ae-mail: prfraser@unimelb.edu.au
} 
(for example $n+{ }^{12} \mathrm{C} \rightarrow{ }^{13} \mathrm{C}[2]$ ) and in exotic targets where the particle-emission widths of states are thin. Indeed, it has been seen to have predictive power, for example, when modelling $p+{ }^{14} \mathrm{O} \rightarrow{ }^{15} \mathrm{~F}[3,4]$.

It is important that the latter be expanded to accommodate cases where particle-emission widths are larger, as in recent years experimental nuclear physics has centred on radioactive beams, and theoretical studies should follow.

\section{Considerations for particle-unstable beams}

In Ref. [5-7], particle-unstable scattering bodies are considered in coupled-channel calculations for heavy mass systems at high energies where resonances are not an important feature in cross sections. However, in the lowenergy and low-mass regime where resonances are important, a different method of accounting for particleinstability is more appropriate: modifying the Green's functions of the $S$-matrix [8]. A complex component is added to the target-state energy, being the experimental width, leading to

$$
\begin{gathered}
{\left[\mathbf{G}_{0}\right]_{n n^{\prime}}=\mu\left[\sum_{c=1}^{\text {open }} \int_{0}^{\infty} \hat{\chi}_{c n}(x) \frac{x^{2}\left[k_{c}^{2}-x^{2}-\frac{i \mu \Gamma_{c}}{2}\right]}{\left[k_{c}^{2}-x^{2}\right]^{2}+\frac{\mu^{2} \Gamma_{c}^{2}}{4}} \hat{\chi}_{c n^{\prime}}(x) d x\right.} \\
\left.-\sum_{c=1}^{\text {closed }} \int_{0}^{\infty} \hat{\chi}_{c n}(x) \frac{x^{2}\left[h_{c}^{2}+x^{2}+\frac{i \mu \Gamma_{c}}{2}\right]}{\left[h_{c}^{2}+x^{2}\right]^{2}+\frac{\mu^{2} \Gamma_{c}^{2}}{4}} \hat{\chi}_{c n^{\prime}}(x) d x\right] .
\end{gathered}
$$

This equation has no poles on the real axis, and integration may proceed normally.

With this change made, resonances in the elastic and total cross sections are broadened and reduced in magnitude, with initially-narrow resonances obscured in the scattering background [8]. In systems so-far studied, compound states widths which are underestimated without considering target-state widths are often over-estimated when these are considered, but in many cases are closer to experimental values [9]. This indicates that there is merit in the approach, but also scope for further development.

With target-state widths included, reaction cross sections are non-zero down to the scattering threshold, which reflects loss of flux due to particle break-up. However, rather than going to zero at the threshold, these show an erroneous asymptotic behaviour.

Another consequence of adding these widths is that for a given parameter set, compound system resonance centroids are moved to a higher energy, and this is problematic in the case of sub-threshold bound states as the widths are only partial and refer to nucleon emission. This problem can be solved by multiplying the widths in the Green's functions by a Heaviside step function. This, however, introduces the new problem of generating two copies of compound system states which are just below the resonance threshold where there are no target widths, but are found to be above when there are [9].

A solution to all problems outlined above is to use target-state widths that have a judiciously-chosen dependence on projectile energy, rather than having a constant value. This, in effect, modifies the shape of the resonant target state such that these problems do not arise.

The idea of introducing energy dependencies is not new to nuclear physics, and goes back to the introduction of penetration factors in the 1950s $[10,11]$. In this case, this introduction takes the form of modifying the Green's functions, viz.

$$
\begin{gathered}
{\left[\mathbf{G}_{0}\right]_{n n^{\prime}}=\mu\left[\sum_{c=1}^{\mathrm{open}} \int_{0}^{\infty} \hat{\chi}_{c n}(x) \frac{x^{2}}{k_{c}^{2}-x^{2}+\frac{i \mu \Gamma_{c} U(E)}{2}} \hat{\chi}_{c n^{\prime}}(x) d x\right.} \\
\left.-\sum_{c=1}^{\text {closed }} \int_{0}^{\infty} \hat{\chi}_{c n}(x) \frac{x^{2}}{h_{c}^{2}+x^{2}-\frac{i \mu \Gamma_{c} U(E)}{2}} \hat{\chi}_{c n^{\prime}}(x) d x\right],
\end{gathered}
$$

where, as minimum conditions, the scaling function $U(E)=0$ when $E \leq 0, U(E)=1$ at energy centroid, and $U(E) \rightarrow 0$ as $E \rightarrow \infty$.

Two possible candidates for $U(E)$ are [12]

$$
\begin{aligned}
& U(E)=\left\{\left[\frac{(1+A)^{2}}{(1+A)^{2}-1}\right]\left[\frac{1}{A\left(1-\frac{E}{E_{r}}\right)^{2}+1}\right]\right. \\
&\left.-\left[\frac{1+A}{(1+A)^{2}-1}\right]\left[\frac{1}{A\left(\frac{E}{E_{r}}\right)^{2}+1}\right]\right\} H(E)
\end{aligned}
$$

and its square,

$$
\begin{aligned}
U(E)=\{ & {\left[\frac{(1+A)^{2}}{(1+A)^{2}-1}\right]\left[\frac{1}{A\left(1-\frac{E}{E_{r}}\right)^{2}+1}\right] } \\
& \left.-\left[\frac{1+A}{(1+A)^{2}-1}\right]\left[\frac{1}{A\left(\frac{E}{E_{r}}\right)^{2}+1}\right]\right\}^{2} H(E)
\end{aligned}
$$

where $E_{r}$ is the centroid energy and $A$ is a parameter.

Another family of candidates is based upon a Wigner distribution [13], modified to meet the necessary conditions:

$$
U(E)=e^{\beta}\left(\frac{E}{E_{r}}\right)^{L} e^{-\beta\left(\frac{E}{E_{r}}\right)^{L}} H(E)
$$

Examples of these equations are shown in Fig. 1, where for Eq. (9) and (10) $A=0.5$, and for Eq. (11) $\beta=1$ and $L=2$.

\section{Preliminary results}

Preliminary investigation of the effects of Eq. (9), only, was reported in Ref. [14] for $n+{ }^{8}$ Be scattering. Elastic and reaction cross sections for this study are reproduced in Fig. 2, along with results for the case where no target-state widths are considered, the 'no-widths' case, the case with target-state widths that are uniform with energy, and scaling using Eq. (10). It was found that anomalous asymptotic behaviour in the reaction cross section is curtailed, but not eliminated, using Eq. (9). However, it is seen that the reaction cross section behaves as required using Eq. (10).

Considering the low- $\frac{E}{E_{r}}$ behaviour of these scaling functions in the inset of of Fig. 1, this suggests that the 


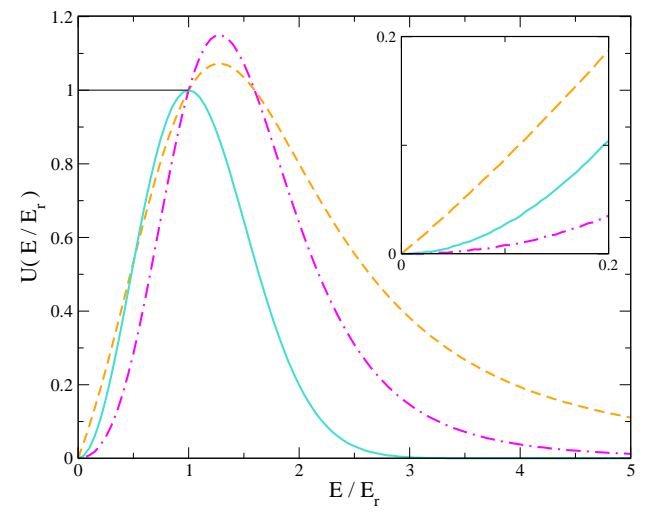

Figure 1. Examples of Eq. (9) (dashed line), Eq. (10) (dotdashed line) and Eq. (11) (solid line), with detail of low- $\frac{E}{E_{r}}$ region.

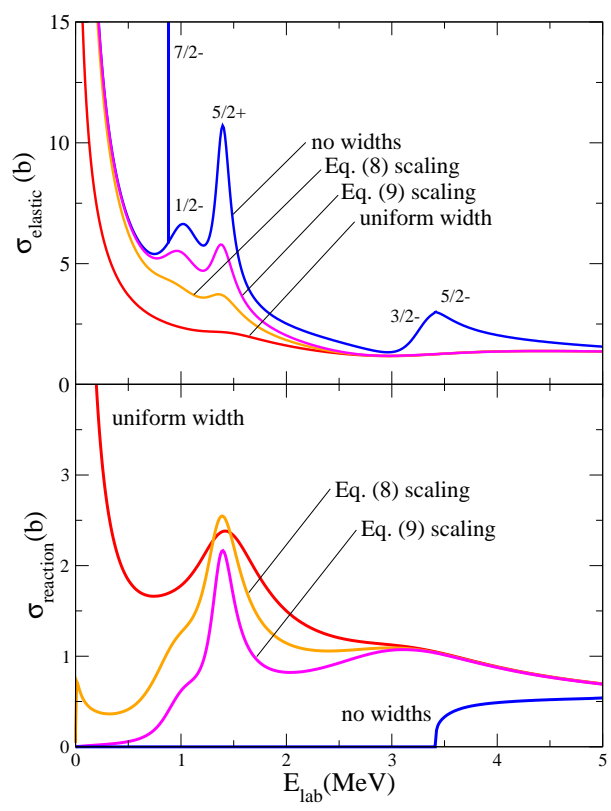

Figure 2. MCAS $n+{ }^{8}$ Be elastic cross sections (upper panel) and reaction cross sections (lower panel) for Eq. (9) and (10).

conditions on the scaling outlined above are not sufficient; in addition, it is required that $\frac{d U(E)}{d E} \rightarrow 0$ as $E \rightarrow+0$.

In Fig. 2 it is seen that at energies below $\sim 3 \mathrm{MeV}$ the influence of target-state widths is reduced, and resonances visible in the no-width case become more prominent. This is more so for the Eq. (9) scaling than that of Eq. (10). Above $\sim 3 \mathrm{MeV}$, the scaled-widths curve closely follows the uniform-widths curve.

Table 1 shows the compound system state widths from experiment, and from theory in the no-width case, the uniform target-state widths case, and the cases where widths are multiplied by Eq. (9) with $A=0.5$, and widths multiplied by Eq. (10) with $A=0.5$, as well as ratios of the theoretical and experimental values. Bold face entries are cases where the calculated values are within a factor of three of experiment. Experimental centroids are shown, along with centroids for the uniform widths calculation, for which the scattering potential parameters are tuned. (The same set was used for all three calculations.)

Focusing on states of ${ }^{9} \mathrm{Be}$ where the centroid is well recreated by theory (being $\frac{1}{2}^{-}, \frac{5}{2}^{+}, \frac{3}{2}^{-}$and $\frac{9}{2}^{+}$), it can be seen that in the no-widths case all underestimate the measured value. For the uniform widths case, all theoretical calculations overestimate measured widths.

In the case of the $\frac{1}{2}^{-}, \frac{5}{2}^{+}$, and $\frac{9}{2}^{+}$states, the results from using both Eq. (9) and Eq. (10) are between those of the underestimated no-widths and over-estimated uniform widths cases, and thus better model experimental data. The $\frac{3}{2}^{-}$results from using Eq. (9) and Eq. (10) both overestimate its width more than that of the uniform widths calculation, but are still in better agreement with data than the no-widths value. These results indicate that this method of including target-state particle-emission widths with a shape modified by a dependence on projectile energy shows promise in cases where MCAS recreates a compound state well.

The upper panel of Fig. 3 shows the elastic cross section that results from using the adjusted Wigner distribution, Eq. (11), as a scaling factor, with parameter values $L=2$ and $\beta=1$. Also shown therein are results of a calculation with no target-state widths considered and a calculation with energy-independent widths included. (N.B. this investigation represents an interaction parameter set which is minutely different from that used in Fig. 2.) As with Fig. 2, below $\sim 3 \mathrm{MeV}$ the influence of target-state widths is reduced, and resonances visible in the no-width case are more pronounced. Above $\sim 3 \mathrm{MeV}$, the scaledwidths curve closely follows the uniform-widths curve.

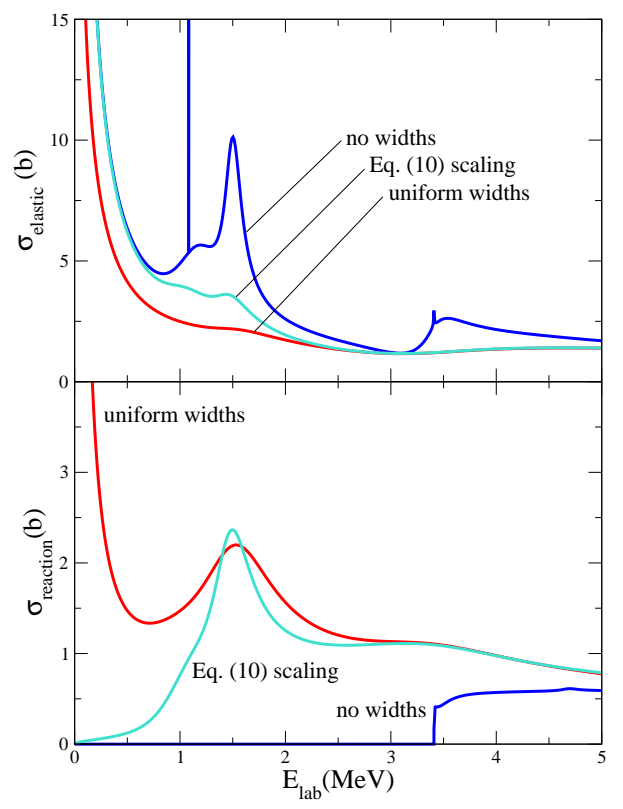

Figure 3. Upper: MCAS $n+{ }^{8}$ Be elastic cross sections (upper panel) and reaction cross sections (lower panel) for Eq. (11).

The lower panel depicts the reaction cross section for the same calculations. The scaled widths curve entirely 
Table 1. Experimental ${ }^{9}$ Be states' $J^{\pi}$, centroids and widths [15]; theoretical widths and ratios to experiment for the case with no target-state widths; theoretical centroids, widths and ratios to experiment for the case with uniform target-state widths; theoretical widths and ratios to experiment for the case where target-state widths vary with Eq. (9); and theoretical widths and ratios to experiment for the case where target-state widths vary with Eq. (10).

\begin{tabular}{ccc|cc|ccc|cc|cc}
\hline \hline$J^{\pi}$ & $E_{\text {exp. }}$ & $\Gamma_{\text {exp. }}$ & $\Gamma_{\text {no-width }}$ & $\frac{\Gamma_{\text {no-width }}}{\Gamma_{\text {exp. }}}$ & $E_{\text {unif. }}$ & $\Gamma_{\text {unif. }}$ & $\frac{\Gamma_{\text {unif. }}}{\Gamma_{\text {exp. }}}$ & $\Gamma_{E q .(8)}$ & $\frac{\Gamma_{E q .(8)}}{\Gamma_{\text {exp. }}}$ & $\Gamma_{E q .(9)}$ & $\frac{\Gamma_{\text {Eq.(9) }}}{\Gamma_{\text {exp. }}}$ \\
\hline$\frac{5}{2}^{-}$ & 0.764 & $7.8 \times 10^{-4}$ & 0.033 & 41.69 & 3.60 & 2.604 & 3338 & 2.712 & 3477 & 2.866 & 3674 \\
$\frac{1}{2}^{-}$ & 1.11 & $1.080 \pm 0.110$ & 0.444 & $\mathbf{0 . 4 1 1}$ & 1.08 & 1.606 & $\mathbf{1 . 4 8 7}$ & 0.801 & $\mathbf{0 . 7 4 2}$ & 0.552 & $\mathbf{0 . 5 1 1}$ \\
$\frac{5}{2}^{+}$ & 1.384 & $0.282 \pm 0.011$ & 0.151 & $\mathbf{0 . 5 3 5}$ & 1.40 & 0.697 & $\mathbf{2 . 4 7 3}$ & 0.378 & $\mathbf{1 . 3 4 2}$ & 0.246 & $\mathbf{0 . 8 7 2}$ \\
$\frac{3}{2}^{+}$ & 3.039 & $0.743 \pm 0.055$ & 4.894 & 6.5868 & 4.88 & 5.294 & 7.125 & 4.538 & 6.108 & 4.026 & 5.419 \\
$\frac{3}{2}^{-}$ & 3.92 & $1.330 \pm 0.360$ & 0.478 & $\mathbf{0 . 3 5 9}$ & 3.39 & 2.930 & $\mathbf{2 . 2 0 3}$ & 3.072 & $\mathbf{2 . 3 1 0}$ & 3.250 & $\mathbf{2 . 4 4 4}$ \\
$\frac{7}{2}^{-}$ & 4.71 & $1.210 \pm 0.230$ & $1.51 \mathrm{E}-5$ & $1.25 \mathrm{E}-5$ & 0.95 & 1.641 & $\mathbf{1 . 3 5 6}$ & 0.373 & 0.308 & 0.090 & 0.074 \\
$\frac{9}{2}^{+}$ & 5.09 & $1.330 \pm 0.090$ & 0.321 & 0.241 & 4.976 & 2.384 & $\mathbf{1 . 7 9 2}$ & 2.080 & $\mathbf{1 . 5 6 4}$ & 1.913 & $\mathbf{1 . 4 3 8}$ \\
\hline \hline
\end{tabular}

eliminates the erroneous behaviour at low energy, due to the shallow gradient of Eq. (11) as $\frac{E}{E_{r}} \rightarrow+0$. Thus, this family of scaling equations warrants further investigation.

\section{Further considerations}

While the above work illustrates the behaviour of several functions that may scale a target state's width as a way of modifying its shape, and will inform the development of this project, the results presented are not final as the formalism is not yet complete. This is because energydependent target-state widths violate causality unless an energy-dependent correction is made to the centroid energy $[12,16,17]$, i.e.,

$$
E_{r}+i \frac{\Gamma}{2} \quad \longrightarrow \quad E_{r}+\Delta E_{r}(E)+i \frac{\Gamma \cdot U(E)}{2}
$$

where

$$
\Delta E_{r}(E)=\frac{1}{\pi} \cdot P \int_{\epsilon_{0}}^{\infty} \frac{U\left(E^{\prime}\right)}{E^{\prime}-E} d E^{\prime} .
$$

\section{Conclusions}

A multi-channel algebraic formalism for $N A$ scattering was briefly reviewed, focusing on how particle-unstable targets are modelled by incorporating particle-emission widths of target states. With these added, cross section resonances are broadened and absorbed into the background, and calculated widths are often closer to experiment. Problems at and below the scattering threshold are currently being solved by introducing energy-dependent target-state widths. Preliminary results from using two families of energy-dependent scaling functions for these widths are often better than in calculations which include no targetstate widths or widths of uniform magnitude.

\section{Acknowledgements}

SK acknowledges support from the National Research Foundation of South Africa. JPS acknowledges support from the National Sciences and Engineering Research Council (NSERC) of Canada. KA thanks the University of Johannesburg, South Africa, for hospitality and support during a recent visit. PRF acknowledges funds from the Dipartimento di Fisica e Astronomia dell'Università di Padova and PRIN research project 2009TWL3MX. RF acknowledges financial support from the Instituto Nacional de Geriatría (project DI-PI-002/2012).

\section{References}

[1] S. Weinberg, in Lectures on Particles and Field Theory (Prentice-Hall, 1965), Vol. Brandeis Summer Institute in Theoretical Physics vol 2., p. 289

[2] K. Amos et al., Nucl. Phys. A728, 65 (2003)

[3] L. Canton et al., Phys. Rev. Lett. 96, 072502 (2006)

[4] I. Mukha et al., Phys. Rev. C 82, 054315 (2010)

[5] W.H.Z. Cárdenas et al., Phys. Rev. C C68, 054614 (2003)

[6] W.H.Z. Cárdenas et al., Braz. J. Phys. 34, 1254 (2004)

[7] P.R.S. Gomes et al., Nucl. Phys. A828, 233 (2009)

[8] P. Fraser et al., Phys. Rev. Lett. 101, 242501 (2008)

[9] P.R. Fraser et al., Rev. Mex. Fís. 57, 20 (2011)

[10] J. Blatt, V. Weisskopf, Theoretical nuclear physics (Dover Publications, New York, 2010)

[11] C. Iliadis, Nuclear physics of stars (Wiley-VCH, Weinheim, 2007)

[12] G. Cattapan et al., Phys. Rev. C 43, 1395 (1991)

[13] M. Mehta, Random Matrices (Academic Press, New York, 1991)

[14] L. Canton et al., Phys. Rev. C 83, 047603 (2011)

[15] D.R. Tilley et al., Nucl. Phys. A745, 155 (2004)

[16] C. Mahaux et al., Nucl. Phys. A A449, 354 (1986)

[17] C. Mahaux et al., Nucl. Phys. A A456, 134 (1986) 